\title{
TREES ARE CONTRACTIBLE
}

\author{
D. G. PAULOWICH
}

\begin{abstract}
Any hereditarily unicoherent, locally connected, compact connected Hausdorff space is contractible using an ordered continuum. An example is given of a hereditarily unicoherent, locally connected, first countable, compact connected Hausdorff space that does not admit the structure of a topological semigroup with zero and identity.
\end{abstract}

1. Definitions and examples. In this note we follow the terminology of [6], where a continuum is a nonempty compact connected Hausdorff space and an arc $[c, d]$ is a continuum with exactly two non-cutpoints $c$ and $d$. Recall also that a space $X$ is defined to be contractible to the point $p \in X$ if there exist both an $\operatorname{arc}[c, d]$ and a continuous function $\phi: X \times[c, d] \rightarrow X$ such that for each $x \in X, \phi(x, c)=p$ and $\phi(x, d)=x$.

By [7, Theorem 9] the continuum $X$ is a tree if and only if $X$ is locally connected and hereditarily unicoherent. Given distinct points $p, q \in X$ there exists a unique arc $[p, q]$ contained in $X$ with endpoints $p$ and $q$. We use $[p, p]$ for $\{p\}$.

It is known that each subcontinuum of a tree is itself a tree. It follows from the main result of this paper that the continuum $X$ is a tree if and only if $X$ is locally connected and each subcontinuum of $X$ is contractible.

We now define a first countable arc $B=[a, b]$. Let $W$ denote the collection of all sequences $\left\{x_{n}\right\}$ of real numbers with the property that only finitely many $x_{n}$ are nonzero and, for each $n, 0 \leqslant x_{n} \leqslant 1$. Let $a \in W$ be the sequence with all entries equal to 0 . Let $b$ be the sequence with all entries equal to 1 . Define " $\leqslant$ " on $B=W \cup\{b\}$ by $\left\{x_{n}\right\} \leqslant\left\{y_{n}\right\}$ if either $x_{n} \leqslant y_{n}$ for all $n$ or there exists a positive integer $n$ such that $x_{n}<y_{n}$ and $x_{m} \leqslant y_{m}$ for all $m>n$.

Let $(B, \leqslant)$ have the order topology. For each $n$, let $b_{n} \in B$ have the first $n$ entries equal to 1 and the remaining entries equal to 0 . Note that the arc $\left[a, b_{n}\right]$ is homeomorphic to $[0,1]^{n}$, with the reverse dictionary order and the order topology.

Proposition. Let $m$ be any positive integer. Let $f:\left[a, b_{m}\right] \rightarrow\left[a, b_{m+1}\right]$ be continuous. For $0 \leqslant t \leqslant 1$, let $A(t)$ denote the set of all $\left\{x_{n}\right\} \in B$ such that $x_{m+1}=t$ and $x_{n}=0$, for all $n \geqslant m+2$. For each $t, A(t)$ is homeomorphic to $A(0)=\left[a, b_{m}\right]$. Then for some $t, f\left(\left[a, b_{m}\right]\right)$ is contained in $A(t)$. Thus if $f(a)=a$, then $f\left(\left[a, b_{m}\right]\right)$ is contained in $\left[a, b_{m}\right]$.

Received by the editors December 9, 1980 and, in revised form, May 29, 1981, and July 7, 1981.

1980 Mathematics Subject Classification. Primary 54F05, 54F50, 54F55.

Key words and phrases. Tree, contractibility, topological semigroup. 
Proof. If $m=1$, we note that the arc $\left[a, b_{1}\right]$ is separable and each $A(t)$ is a maximal separable subarc of $\left[a, b_{2}\right]$. We now use induction to prove the proposition when $m \geqslant 2$. We may assume that the following result has already been established: if the function $g:\left[a, b_{m-1}\right] \rightarrow\left[a, b_{m}\right]$ is continuous then, for some $t, g\left(\left[a, b_{m-1}\right]\right)$ is contained in $B(t)$, where $B(t)$ is the set of all $\left\{x_{n}\right\} \in B$ such that $x_{m}=t$ and $x_{n}=0$, for all $n \geqslant m+1$.

Define $H:\left[a, b_{m+1}\right] \rightarrow\left[a, b_{m}\right]$ by setting $H\left(\left\{x_{n}\right\}\right)=\left\{y_{n}\right\}$, where $y_{n}=x_{n+1}$, for $1 \leqslant n \leqslant m$, and $y_{n}=0$, for all $n \geqslant m+1$. Now $H$ is continuous and for each $\left\{y_{n}\right\}$, $H^{-1}\left(\left\{y_{n}\right\}\right)$ is a maximal separable subarc of $\left[a, b_{m+1}\right]$. We note that $H^{-1}(B(t))=$ $A(t)$, for $0 \leqslant t \leqslant 1$.

Let $h:\left[a, b_{m}\right] \rightarrow\left[a, b_{m-1}\right]$ be the restriction of $H$ to $\left[a, b_{m}\right]$. Since the image of a separable space is itself separable, there exists a continuous function $g:\left[a, b_{m-1}\right] \rightarrow$ $\left[a, b_{m}\right]$ such that $(g \circ h)=(H \circ f)$. By assumption, we conclude that $g\left(\left[a, b_{m-1}\right]\right)$ is contained in $B(t)$, for some $0 \leqslant t \leqslant 1$. But then $f\left(\left[a, b_{m}\right]\right)$ is contained in $H^{-1}(B(t))$ $=A(t)$.

Let $f: B \rightarrow B$ be continuous. By the Proposition, if $f(a)=a$ then $f\left(\left[a, b_{m}\right]\right)$ is contained in $\left[a, b_{m}\right]$, for all $m$. Another consequence of the Proposition is that $f(a)=b$ implies $f$ is constant.

Now $B=[a, b]$ is a remarkable continuum which can be used to construct other spaces of interest. Let $P$ be the first countable continuum obtained by identifying the non-cutpoints of $B$. The hyperspace of all subcontinua of $P$ is not a contractible space, by a proof similar to that of [6, Theorem 5].

It is known [3, Theorem $\mathrm{A}$ ] that each metric tree admits the structure of a topological semilattice with zero and identity. It follows that each metric tree is contractible (using a metric arc). Eberhart [1] gives an example of a nonmetrizable tree which does not admit the structure of a topological semigroup with zero and identity. We modify his work to produce a first countable example.

Let $S=\{1,2,3,4,5,6\}$ have the discrete topology and let $X$ be the space obtained from $B \times S$ by identifying:

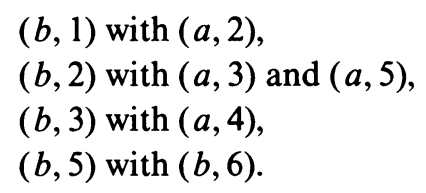

Let $\psi: B \times S \rightarrow X$ be the identification map. Let $q_{n}=\psi(b, n), 1 \leqslant n \leqslant 5$, and let $q_{0}=\psi(a, 1), q_{6}=\psi(a, 6)$. Then the continuum $X$ is a first countable tree with exactly three non-cutpoints $q_{0}, q_{4}$, and $q_{6}$. We claim that $X$ is the desired example.

Suppose not, i.e. there is a continuous multiplication $\phi: X \times X \rightarrow X$ with zero $p \in X$ and identity $q \in X$. By [2, Exercise 17, p. 169], the identity element $q$ must be a non-cutpoint of $X$. We sketch the proof for the case $q=q_{4}$ (the other two cases being handled in a similar manner).

Suppose $p=q_{0}$. Let $x=q_{6}$ and define the retraction map $r: X \rightarrow[p, x]$ by 
$r(y)=y$, if $y \in[p, x]$, and $r(y)=q_{2}$, if $y \in\left[q_{2}, q\right]$. Define $F:[p, q] \rightarrow[p, x]$ by $F(z)=(r \circ \phi)(x, z)$, for each $z \in[p, q]$. Then $F(p)=p$ and $F(q)=x$. But the function $F$ is continuous and $\left[q_{0}, q_{1}\right]$ is homeomorphic to $B$. By the Proposition, $F\left(\left[q_{0}, q_{1}\right]\right)$ is contained in $\left[q_{0}, q_{1}\right]$. At best we have $F\left(q_{1}\right)=q_{1}$ and then (by the Proposition) $F\left(\left[q_{1}, q_{2}\right]\right)$ is contained in $\left[q_{0}, q_{2}\right]$. At best we have $F\left(q_{2}\right)=q_{2}$ and then (by the Proposition) $F\left(\left[q_{2}, q_{3}\right]\right)$ is contained in $\left[q_{0}, q_{5}\right]$. At best we have $F\left(q_{3}\right)=q_{5}$. But $\left[q_{2}, q_{6}\right]$ is homeomorphic to the union of two copies of $B$, meeting at the point $b$ in each arc. It is then a consequence of the Proposition that $F\left(\left[q_{3}, q_{4}\right]\right)$ equals $\left\{q_{5}\right\}$. We conclude that we always have $F\left(\left[q_{0}, q_{4}\right]\right)$ contained in $\left[q_{0}, q_{5}\right]$ and thus $F(q)=$ $F\left(q_{4}\right)$ is not equal to $x=q_{6}$. A contradiction has been reached. Essentially the same proof works for any point $p \in\left[q_{0}, q_{4}\right]$.

Suppose $p \in\left[q_{2}, q_{6}\right]$. Then by choosing a retraction map $r: X \rightarrow[p, x]$, where $x=q_{0}$, and defining $F:[p, q] \rightarrow[p, x]$ by $F(z)=(r \circ \phi)(x, z)$, we can again use the Proposition to reach the contradictory conclusion: $F(q)$ is not equal to $x$. This ends the proof for the case $q=q_{4}$.

We note that the preceding proof also shows that there is no subarc $[p, q]$ of $X$ such that $X$ is contractible to the point $p \in X$, using the $\operatorname{arc}[p, q]$.

2. Proof of main result. Let $T$ be any tree containing more than one point and let $C(T)$ be the hyperspace of all subcontinua of $T$ with the finite topology [5]. We shall prove that, for each $t \in T$, the space $T$ is contractible to the point $t$ using some arc $[c, d]$ contained in the hyperspace $C(T)$. We note that hyperspaces are arcwise connected [4]. For each subcontinuum $A \in C(T)$ there is a unique continuous retraction $h_{A}: T \rightarrow A$ such that, for each $x \in A,\left\{t \in T: h_{A}(t)=x\right\}$ is a continuum. Define $h: T \times C(T) \rightarrow T$ by, for each $t \in T$ and each $A \in C(T), h(t, A)=h_{A}(t)$. We note that for all $s, t \in T$ we have $h(s,\{t\})=t$ and $h(s, T)=s$. Given the fact that there is an arc in the hyperspace joining $\{t\}$ and $T$, we have only to prove that the function $h$ is continuous.

Consider a net $\left\{\left(t_{n}, A_{n}\right): n \in \Gamma\right\}$ converging to $(t, A)$ in $T \times C(T)$, such that the net $\left\{h\left(t_{n}, A_{n}\right): n \in \Gamma\right\}$ converges to $y \in T$. We show that $y=h(t, A)$.

Let $U$ be any open set containing $A$. Then there exists an element $m$ such that, for all $n \geqslant m, A_{n} \in U$. Thus $h\left(t_{n}, A_{n}\right) \in U$. Therefore $y$ is in the closure of $U$. We conclude that $y \in A$.

Let $q \in A, q \neq h(t, A)$. Choose a cutpoint $p$ in the arc $[q, h(t, A)]$. Let $V$ be the component of $(X-\{p\})$ containing $h(t, A)$. Let $U$ be the component of ( $X-$ $\{h(t, A)\})$ containing $p$. Then $U, V$ are open sets and $t \in V$. Let $\langle U, V\rangle$ denote the ngbhd of $A$ in the hyperspace consisting of all subcontinua $K \subset(U \cup V)$ having nonempty intersection with both $U$ and $V$. Then there exists an element $m$ such that, for all $n \geqslant m$, we have $t_{n} \in V$ and $A_{n} \in\langle U, V\rangle$. Thus there exists a point $x_{n} \in\left(A_{n}\right.$ $\cap V)$. But then $h\left(t_{n}, A_{n}\right)$ is contained in $\left[t_{n}, x_{n}\right]$ which is contained in $V$. Therefore $y$ is in the closure of $V$ and so $y \neq q$. We conclude that $y=h(t, A)$. This completes the proof.

The author wishes to thank Professor Michael Edelstein for suggesting the use of nets in the preceding proof. 


\section{REFERENCES}

1. Carl Eberhart, Some classes of continua related to clan structures, dissertation, Louisiana State University, 1966.

2. K. H. Hofmann and P. S. Mostert, Elements of compact semigroups, Merrill, Columbus, 1966.

3. R. J. Koch and L. F. McAuley, Semigroups on continua ruled by arcs, Fund. Math. 56 (1964), 1-8.

4. M. M. McWaters, Arcs, semigroups, and hyperspaces, Canad. J. Math. 20 (1968), 1207-1210.

5. E. Michael, Topologies on spaces of subsets, Trans. Amer. Math. Soc. 71 (1951), 152-182.

6. D. G. Paulowich, Weak contractibility and hyperspaces, Fund. Math. 94 (1977), 41-47.

7. L. E. Ward, Jr., Mobs, trees, and fixed points, Proc. Amer. Math. Soc. 8 (1957), 798-804.

Department of Mathematics, Dalhousie University, Halifax, Nova Scotia, Canada B3H 4H8

Current address: Department of Mathematics, Saint Mary's University, Halifax, Nova Scotia, Canada B3H 3C3 